\title{
Garcinia Mangostana Extract and Curcumin Ameliorate Oxidative Stress, Dyslipidemia, and Hyperglycemia in High Fat Diet-induced Obese Wistar Albino Rats
}

\author{
Ranyah Labban \\ King Saud University \\ Hanan fawaz \\ King Saud University \\ Ahmed Almnaizel \\ King Saud University \\ May Al-Muammar \\ King Saud University \\ Ramesa Bhat \\ King Saud University \\ Afaf El-Ansary ( $\square$ elansary@ksu.edu.sa ) \\ King Saud University
}

\section{Research Article}

Keywords: Garcinia mangostana, Curcuma, Obesity, High fat diet, Oxidative stress, dyslipdemia, hyperglycemia

Posted Date: January 15th, 2021

DOI: https://doi.org/10.21203/rs.3.rs-122559/v1

License: (c) (i) This work is licensed under a Creative Commons Attribution 4.0 International License. Read Full License

Version of Record: A version of this preprint was published at Scientific Reports on March 31st, 2021. See the published version at https://doi.org/10.1038/s41598-021-86545-z. 


\section{Abstract}

Background:

Obesity is a risk factor for several diseases related to oxidative stress, hyperlipidemia, and hyperglycemia. Several pharmacological agents have been used to treat obesity, but these commonly exhibit undesirable side effects. The aim of this study was to explore the effects of Garcinia mangostana (mangosteen) and Curcuma longa independently and synergistically in modulating selected biochemical markers of oxidative stress, dyslipidemia, and hyperglycemia commonly observed in high-fat diet-induced obesity in rodent models.

Methods:

Male albino Wistar rats were divided into eight experimental groups, fed on a normal diet or high-fat diet (HFD), then given mangosteen extract ( $400 \mathrm{mg} / \mathrm{kg}$ body weight/day) and/or curcumin $(80 \mathrm{mg} / \mathrm{kg}$ body weight/day) for 6 weeks. Oxidative stress markers, glucose, and lipid fractions were measured in the sera.

Results:

Curcumin was found to be more effective in reducing $\mathrm{BMI}$, while mangosteen extract was found to induce a significant increase in anti-atherogenic marker HDL-C, but was ineffective in reducing dyslipidemia. Mangosteen extract and curcumin effectively reduced blood glucose. Mangosteen did not exhibit any anti-oxidative effects in normal weight rats; however, it induced a significant increase in glutathione in obese rats.

Conclusion:

The present study demonstrated that mangosteen pericarp extract and curcumin were independently and synergistically effective in treating obesity-induced atherogenesis.

\section{Introduction}

Obesity is a complex metabolic disorder with multifactorial etiology. Obesity can lead to reduced quality of life due to numerous associated complications, including cardiovascular disease, diabetes, cancer, asthma, sleep disorders, liver and renal dysfunction, and sterility. Increasing evidence suggests that oxidative stress plays a critical role in linking obesity to these associated complications[1-3].

Recent studies have proposed that the causes of oxidative stress in obesity include hyperleptinemia, hyperglycemia, insufficient antioxidant capacity, increased free radical generation, mitochondrial dysfunction, and chronic inflammation [4,5]. Accumulating evidence also shows that the quality of diet has demonstrated a negative shift toward consumption of high-energy foods, while intake of nutrient-rich foods such as fruits and vegetables has decreased [6,7]. Such low-quality diets have been linked to a higher risk of obesity (Azizi, 2017); consequently, dietary modification is frequently recommended as the 
main approach in prevention and treatment of obesity $[8,9]$. Many studies have also indicated that natural products such as phytochemicals in fruits and vegetables can be vital modulators of the risks accompanying obesity.

Mangosteen (Garcinia mangostana L.) fruit has a unique sweet-sour taste, and is rich in valuable compounds, such as xanthones. Mangosteen has been used in various traditional remedies to treat fever, diarrhea, and to promote wound healing. Recently, it has been used as a chief constituent in health supplements for weight loss and for supporting overall health [10], due to its well-known anti-oxidative and anti-inglammatory medicinal properties [11-13]. Mangosteen pericarp contains mostly sugars (nearly $50 \%$ of total metabolites), followed by additional metabolites such as organic acids, alcohols, sugar acids, and aromatic compounds. Additionally, mangosteen pericarp contains several phenolic compounds, such as benzoic acid, tyrosol, and protocatechuic acid, which are known to have antioxidative and anti-inflammatory effects.

Interestingly, various studies have revealed that spices such as curcumin may show efficacy in controlling weight gain or reducing obesity. Curcumin is a bioactive polyphenol component found in turmeric rhizomes [14]. Clinical effects of curcumin in reducing weight and body mass index (BMI) have not been thoroughly assessed, and the results of many studies are unreliable and contradictory. Multiple studies have reported that a bioavailable form of curcumin resulted in improved weight management in obese humans and experimental animals $[15,16]$.

High-energy diets have been utilized to induce obesity and related metabolic disorders in rodent models; however, dietary mediation has not been absolutely standardized [17]. Usually, these diets comprise a simple exchange of carbohydrate-derived calories with fat-derived calories and are compared with a standard chow diet (SCD) as a control.

In this study, we sought to determine the potency of Garcinia mangostana pericarp extract and curcumin either independently or synergistically in modulating biochemical markers of oxidative stress, hyperlipidemia, and hyperglycemia in a high-fat diet-induced rodent model of obesity.

\section{Methods}

\subsection{Preparation of mangosteen extract}

Mangosteen fruits were obtained from a local market in Riyadh, and authenticated by a taxonomist from the Department of Botany, College of Science, King Saud University, Riyadh. $500 \mathrm{~g}$ of the dried pericarp of mangosteen was soaked in 1.5 liters of $99.7 \%$ methanol at room temperature for $72 \mathrm{~h}$. Extracts were filtered through Whatman No. 1 filter paper, and solvent was evaporated using a rotary evaporator at $60^{\circ} \mathrm{C}$. The resulting viscous concentrate was freeze-dried to ensure complete removal of solvent, and stored at $-20^{\circ} \mathrm{C}$ for further use. 


\subsection{Preparation of curcumin}

Fresh curcumin was obtained from a local market in Riyadh, and dried after proper cleaning using a drying oven with an air fan at $50^{\circ} \mathrm{C}$ for $3-5 \mathrm{~h}$. Curcumin was then ground and sieved, and the remaining soft powder was stored at $-80^{\circ} \mathrm{C}$ until further use.

\subsection{Animals}

Eighty male albino Wistar rats, twenty-one days old and weighing approximately 60 to $70 \mathrm{~g}$, were obtained from the Experimental Surgery and Animal Laboratory of the Prince Naif Health Research Center (PNHRC). The animals were housed under normal laboratory conditions. All experiments were performed in accordance with the guidelines of the National Institutes of Health for the Care and Use of Laboratory Animals and approved by the Animal Ethics Committee of King Saud University (reference no: KSU-SE-1817).

\subsection{Animal groups and diets}

Animals were divided into eight groups of 5 animals each. Group 1 serves as the control group, as animals were fed a normal diet. Group 2 received a normal diet and $400 \mathrm{mg} / \mathrm{kg}$ body weight/day water extract of mangosteen pericarp for 6 weeks. Group 3 rats received a normal diet and curcumin at 80 $\mathrm{mg} / \mathrm{kg}$ body weight/day for 6 weeks. Group 4 received a normal diet and both mangostine pericarp extract ( $400 \mathrm{mg} / \mathrm{kg}$ body weight/day) and curcumin ( $80 \mathrm{mg} / \mathrm{kg}$ body weight/day) for 6 weeks. Group 5 was fed with a high-fat diet (HFD) for 4 weeks. Group 6 rats were fed a HFD for 4 weeks followed by 400 $\mathrm{mg} / \mathrm{kg}$ body weight/day water extract of mangosteen pericarp for 6 weeks. Group 7 received HFD for 4 weeks, followed by $80 \mathrm{mg} / \mathrm{kg}$ body weight/day curcumin for 6 weeks. Group 8 animals received a HFD for 4 weeks, followed by both mangosteen pericarp extract $(400 \mathrm{mg} / \mathrm{kg}$ body weight/day) and curcumin ( $80 \mathrm{mg} / \mathrm{kg}$ body weight/day) for 6 weeks. At the end of the experiment, animals were anesthetized with $5.0 \%$ of sevoflurane and $100 \%$ oxygen, the flow rate of sevoflurane was determined as the following formula: Flow rate $(\mathrm{ml} / \mathrm{min})=0.5 \times$ body weight $(\mathrm{g})$. Blood was collected from the eye and heart using hematocrit capillaries $(75 \mathrm{~mm} / 75 \mu \mathrm{l})$. Our study was carried out in compliance with the ARRIVE guidelines.

\subsection{Collection of serum}

Serum was collected from blood samples by centrifugation for $15 \mathrm{~min}$ at $4,000 \mathrm{rpm}$. Serum was further utilized in the analysis of the following biochemical parameters.

\subsubsection{Levels of serum lipid fractions}


Cholesterol, triglyceride, HDL, and LDL levels were quantitatively determined using commercial kits from United Diagnostics Industry.

\subsubsection{Levels of serum glucose}

Quantitative determination of glucose levels was made via the use of Trinder glucose oxidase I activity test using a kit from, a product of United Diagnostics Industry Company.

\subsubsection{Level of serum Oxidative stress markers}

Lipid oxidation was estimated according to the method reported by Begonaruizlarrea, Maleal, Liza, Lacort \& Degroot [18]. Vitamin C was estimated using the method described by Jagota \& Dani [19]. Glutathione levels were evaluated per the method described by Beutler, Duron \& Kelly [20]. Glutathione S-transferase (GST) activity was determined by method reported by [21].

\subsection{Statistical analysis}

Data are expressed as means \pm standard deviation (SD). To compare results between groups, one-way analysis of variance (ANOVA) tests were used. Significance was assigned at the level of $p<0.05$. The receiver operating characteristics curve (ROC) was analyzed, and the area under the curve (AUC), cutoff values, and degrees of sensitivity and specificity were calculated.

\section{Results}

Body weight, weight gain, and BMI in normal and HFD-induced obese rats in response to mangosteen extract and curcumin are shown in Table 1. There was a significant difference between normal and obese experimental groups in weight $(p \otimes 0.001)$. While the BMI in obese controls (Group 5) reached $0.86 \pm 0.09$ $\mathrm{gm} / \mathrm{cm}^{2}$, the normal diet controls (Group 1) recorded a significantly lower BMI of $0.55 \pm 0.04 \mathrm{gm} / \mathrm{cm}^{2}(p \otimes$ 0.001). Both mangosteen extract and curcumin ameliorated the increase in BMI as measure of obesity; while mangosteen pericarp extract induced a remarkable decrease in BMI (from 0.86 to $0.81 \mathrm{gm} / \mathrm{cm}^{2}$ ), curcuma either alone or in combination was more effective, as BMls were reduced to 0.78 and 0.79 $\mathrm{gm} / \mathrm{cm}^{2}$, respectively.

Levels of malondialdehyde (MDA) serve as a marker of lipid peroxidation and oxidative stress. Glutathione (GSH) levels, GST activity, and vitamin C levels also serve as markers of anti-oxidative capacity. Unexpectedly, obese controls did not show any significant increase in MDA levels or decrease in GSH levels, GST activity, or vitamin C levels (Table 2). While mangosteen pericarp extract did not affect these antioxidant markers in normal weight rats, it induced a significant increase of GSH in obese rats $(123.86 \pm 15.53 \mu \mathrm{g} / \mathrm{ml}$ vs $288.72 \pm 121.37 \mu \mathrm{g} / \mathrm{ml})$. There was also a significant increase in GST activity in rats treated with both mangosteen extract and curcumin ( $p \otimes 0.016)$, although independently these 
compounds did not achieve a significant effect. On the other hand, vita min C levels did not demonstrate any change either in HFD or in treated animals (Table 2).

Dyslipidemia was measured by blood cholesterol, TAG, LDL-C, and HDL-C levels. Obese rats displayed significantly higher cholesterol, TAG, and LDL-C levels, and non-significantly lower HDL-C levels (Table 3). While mangosteen pericarp extract was ineffective in reducing dyslipidemia-related markers (total and LDL-C), it did induce a significant increase in HDL-C, an anti-atherogenic marker ( $p \otimes 0.026)$.

Obese rats also had higher total cholesterol/HDL-C and HDL-C/LDL-C values, clinically used measures that indicate a risk factor for cardiovascular disease. While normal weight rats recorded ratios of $1.52 \pm$ 0.28 and $4.62 \pm 1.21$, respectively, obese rats recorded much higher total cholesterol/HDL-C (3.02 \pm 0.78$)$ concomitant with much lower HDL-C/LDL-C $(1.15 \pm 0.73)$ ratios (Table 4). Serum glucose was also measured in the eight experimental groups. While HFD-induced obesity did not lead to elevation of blood glucose, mangosteen pericarp extract was effective in reducing glucose levels in both normal weight and obese animals (Table 4).

Correlations between all measured markers were also calculated. Negative correlations were observed between BMI, antioxidant status (GSH levels), and HDL-cholesterol (Table 5). BMI was also positively correlated with dyslipidemia-related markers CHOL, LDL, TAG, and CHOL/HDL-C.

ROC and AUC were calculated to measure specificity and sensitivity in the four obese groups. Among the measured variables, BMI was the most efficient variable either to predict obesity in HFD-fed mice or the therapeutic potency of curcumin and mangosteen (Table 6). The other measured variables demonstrated fair $(0.5-0.65)$, good $(0.7-0.85)$, and excellent $(0.85-1)$ predictive values with relative specificity and sensitivity (Table 6).

Multiple regression analysis was also performed using GSH levels as the dependent variable. Interestingly, we observed that MDA as measure of oxidative stress together with dyslipidemia-related independent variables greatly contributed to GSH depletion. 
Table 1

Independent and synergistic effects of curcumin and mangosteen extracts on BMI of lean and HFDinduced obese rats.

\begin{tabular}{|c|c|c|c|c|}
\hline Parameters & Groups & Mean \pm SD & p-value a & $p$-value b \\
\hline \multirow{8}{*}{$\begin{array}{l}\text { BW } \\
\text { (g) }\end{array}$} & Control lean & $306.80 \pm 32.38$ & & \\
\hline & Mangosteen lean & $341.00 \pm 70.84$ & & 0.355 \\
\hline & Curcumin lean & $360.00 \pm 52.56$ & & 0.090 \\
\hline & Synergistic lean & $317.00 \pm 15.67$ & & 0.544 \\
\hline & Control obese & $528.40 \pm 33.92$ & 0.001 & \\
\hline & Mangosteen obese & $504.00 \pm 14.85$ & 0.006 & 0.179 \\
\hline & Curcumin obese & $524.00 \pm 21.63$ & 0.001 & 0.813 \\
\hline & Synergistic obese & $464.00 \pm 38.19$ & 0.001 & 0.023 \\
\hline \multirow{8}{*}{$\begin{array}{l}\text { Weight gain } \\
\text { (g) }\end{array}$} & Control lean & $152.00 \pm 30.83$ & & \\
\hline & Mangosteen lean & $210.80 \pm 72.81$ & & 0.153 \\
\hline & Curcumin lean & $207.20 \pm 46.40$ & & 0.058 \\
\hline & Synergistic lean & $207.00 \pm 13.25$ & & 0.006 \\
\hline & Control obese & $458.60 \pm 35.66$ & 0.001 & \\
\hline & Mangosteen obese & $405.00 \pm 16.67$ & 0.003 & 0.016 \\
\hline & Curcumin obese & $411.20 \pm 21.05$ & 0.001 & 0.034 \\
\hline & Synergistic obese & $392.00 \pm 38.63$ & 0.001 & 0.022 \\
\hline \multirow{8}{*}{$\begin{array}{l}\text { BMI } \\
\left(\mathrm{g} / \mathrm{cm}^{2}\right)\end{array}$} & Control lean & $0.55 \pm 0.04$ & & \\
\hline & Mangosteen lean & $0.61 \pm 0.06$ & & 0.102 \\
\hline & Curcumin lean & $0.65 \pm 0.09$ & & 0.065 \\
\hline & Synergistic lean & $0.57 \pm 0.02$ & & 0.423 \\
\hline & Control obese & $0.86 \pm 0.09$ & 0.001 & \\
\hline & Mangosteen obese & $0.81 \pm 0.06$ & 0.001 & 0.360 \\
\hline & Curcumin obese & $0.78 \pm 0.03$ & 0.030 & 0.105 \\
\hline & Synergistic obese & $0.79 \pm 0.07$ & 0.001 & 0.259 \\
\hline
\end{tabular}


a Significant difference between normal weight and obese rats in each group (Control, Mangosteen, Curcumin, and Synergistic)

b Significant difference between each group and respective normal weight or obese control groups 
Table 2

Independent and synergistic effects of curcumin and mangosteen extract on oxidative stress- and antioxidant-related markers in lean and HFD-induced obese rats.

\begin{tabular}{|c|c|c|c|c|}
\hline \multicolumn{5}{|l|}{ Parameters } \\
\hline & Groups & Mean \pm SD & $p$-value a & $p$-value $b$ \\
\hline \multirow{8}{*}{$\begin{array}{l}\text { MDA } \\
(\mu \mathrm{g} / \mathrm{ml})\end{array}$} & Control lean & $108.95 \pm 12.54$ & & \\
\hline & Mangosteen lean & $100.18 \pm 0.98$ & & 0.193 \\
\hline & Curcumin lean & $94.77 \pm 8.12$ & & 0.067 \\
\hline & Synergistic lean & $93.17 \pm 11.36$ & & 0.071 \\
\hline & Control obese & $94.97 \pm 13.70$ & 0.131 & \\
\hline & Mangosteen obese & $81.21 \pm 23.90$ & 0.114 & 0.296 \\
\hline & Curcumin obese & $94.73 \pm 24.01$ & 0.997 & 0.984 \\
\hline & Synergistic obese & $101.98 \pm 9.61$ & 0.222 & 0.377 \\
\hline \multirow{8}{*}{$\begin{array}{l}\text { GSH } \\
(\mu \mathrm{g} / \mathrm{ml})\end{array}$} & Control lean & $148.31 \pm 36.42$ & & \\
\hline & Mangosteen lean & $123.86 \pm 15.53$ & & 0.205 \\
\hline & Curcumin lean & $125.11 \pm 16.23$ & & 0.230 \\
\hline & Synergistic lean & $162.51 \pm 47.20$ & & 0.609 \\
\hline & Control obese & $168.50 \pm 20.49$ & 0.312 & \\
\hline & Mangosteen obese & $288.72 \pm 121.37$ & 0.017 & 0.047 \\
\hline & Curcumin obese & $140.94 \pm 8.25$ & 0.088 & 0.024 \\
\hline & Synergistic obese & $152.84 \pm 36.07$ & 0.726 & 0.423 \\
\hline \multirow{8}{*}{$\begin{array}{l}\text { GST activity } \\
(\mu / m \mathrm{l})\end{array}$} & Control lean & $1.35 \pm 0.42$ & & \\
\hline & Mangosteen lean & $1.73 \pm 0.39$ & & 0.115 \\
\hline & Curcumin lean & $1.23 \pm 0.27$ & & 0.599 \\
\hline & Synergistic lean & $0.68 \pm 0.86$ & & 0.249 \\
\hline & Control obese & $1.64 \pm 1.46$ & 0.600 & \\
\hline & Mangosteen obese & $2.77 \pm 2.33$ & 0.599 & 0.346 \\
\hline & Curcumin obese & $1.37 \pm 0.23$ & 0.293 & 0.465 \\
\hline & Synergistic obese & $2.39 \pm 0.53$ & 0.016 & 0.117 \\
\hline Vit. C & Control lean & $0.04 \pm 0.01$ & & \\
\hline
\end{tabular}




\begin{tabular}{|llll|}
\hline$\mu \mathrm{g} / \mathrm{ml})$ & Mangosteen lean & $0.05 \pm 0.03$ & 0.602 \\
\cline { 2 - 3 } & Curcumin lean & $0.07 \pm 0.09$ & 0.675 \\
\hline Synergistic lean & $0.03 \pm 0.01$ & 0.094 \\
\hline Control obese & $0.03 \pm 0.00$ & 0.075 & \\
\hline Mangosteen obese & $0.02 \pm 0.01$ & 0.016 & 0.115 \\
\hline Curcumin obese & $0.06 \pm 0.08$ & 0.141 & 0.462 \\
\hline Synergistic obese & $0.03 \pm 0.01$ & 0.402 & 0.600 \\
\hline & & & \\
a Significant difference between normal weight and obese rats in each group (Control, Mangosteen, \\
Curcumin, and Synergistic) \\
b Significant difference between each group and respective normal weight or obese control groups \\
\hline
\end{tabular}


Table 3

Independent and synergistic effects of curcumin and mangosteen extract on the lipid profiles of lean and HFD-induced obese rats.

\begin{tabular}{|c|c|c|c|c|}
\hline Parameters & Groups & Mean \pm SD & $\mathrm{p}$-value a & $p$-value $b$ \\
\hline \multirow[t]{8}{*}{ CHOL (mg/dl) } & Control lean & $74.26 \pm 6.97$ & & \\
\hline & Mangosteen lean & $105.51 \pm 14.21$ & & 0.002 \\
\hline & Curcumin lean & $104.07 \pm 9.84$ & & 0.001 \\
\hline & Synergistic lean & $88.87 \pm 2.93$ & & 0.003 \\
\hline & Control obese & $148.00 \pm 48.24$ & 0.026 & \\
\hline & Mangosteen obese & $182.22 \pm 9.31$ & 0.001 & 0.189 \\
\hline & Curcumin obese & $123.41 \pm 34.60$ & 0.287 & 0.381 \\
\hline & Synergistic obese & $145.97 \pm 21.93$ & 0.004 & 0.934 \\
\hline \multirow[t]{8}{*}{$\mathrm{HDL}-\mathrm{C}(\mathrm{mg} / \mathrm{dl})$} & Control lean & $54.95 \pm 8.71$ & & \\
\hline & Mangosteen lean & $72.29 \pm 17.96$ & & 0.088 \\
\hline & Curcumin lean & $56.18 \pm 11.46$ & & 0.853 \\
\hline & Synergistic lean & $68.57 \pm 18.27$ & & 0.171 \\
\hline & Control obese & $51.45 \pm 17.76$ & 0.702 & \\
\hline & Mangosteen obese & $55.08 \pm 8.81$ & 0.026 & 0.693 \\
\hline & Curcumin obese & $50.16 \pm 12.30$ & 0.446 & 0.897 \\
\hline & Synergistic obese & $50.94 \pm 13.88$ & 0.124 & 0.961 \\
\hline \multirow[t]{8}{*}{ LDL-C (mg/dl) } & Control lean & $11.12 \pm 3.89$ & & \\
\hline & Mangosteen lean & $31.88 \pm 12.21$ & & 0.016 \\
\hline & Curcumin lean & $30.30 \pm 10.07$ & & 0.009 \\
\hline & Synergistic lean & $13.19 \pm 3.25$ & & 0.463 \\
\hline & Control obese & $65.05 \pm 43.59$ & 0.075 & \\
\hline & Mangosteen obese & $95.94 \pm 8.81$ & 0.009 & 0.173 \\
\hline & Curcumin obese & $49.35 \pm 16.88$ & 0.076 & 0.602 \\
\hline & Synergistic obese & $58.45 \pm 15.91$ & 0.009 & 0.754 \\
\hline \multirow[t]{2}{*}{ TAG $(\mathrm{mg} / \mathrm{dl})$} & Control lean & $182.29 \pm 31.89$ & & \\
\hline & Mangosteen lean & $194.60 \pm 24.94$ & & 0.516 \\
\hline
\end{tabular}




\begin{tabular}{llll} 
Curcumin lean & $196.49 \pm 18.04$ & & 0.411 \\
\hline Synergistic lean & $215.54 \pm 6.71$ & & 0.052 \\
\hline Control obese & $223.07 \pm 14.77$ & 0.032 & \\
\hline Mangosteen obese & $222.53 \pm 3.91$ & 0.038 & 0.941 \\
\hline Curcumin obese & $197.22 \pm 89.32$ & 0.987 & 0.556 \\
\hline Synergistic obese & $250.27 \pm 44.94$ & 0.126 & 0.234
\end{tabular}

a Significant difference between normal weight and obese rats in each group (Control, Mangosteen, Curcumin, and Synergistic)

b Significant difference between each group and respective normal weight or obese control groups 
Table 4

Independent and synergistic effects of curcumin and mangosteen extract on cardiovascular risk factors in lean and HFD-induced obese rats.

\begin{tabular}{|c|c|c|c|c|}
\hline Parameters & Groups & Mean \pm SD & $\mathrm{p}$-value a & $p$-value $b$ \\
\hline \multirow[t]{8}{*}{$\mathrm{CHOL} / \mathrm{HDL}-\mathrm{C}$} & Control lean & $1.52 \pm 0.28$ & & \\
\hline & Mangosteen lean & $1.49 \pm 0.24$ & & 0.877 \\
\hline & Curcumin lean & $1.73 \pm 0.40$ & & 0.352 \\
\hline & Synergistic lean & $1.36 \pm 0.32$ & & 0.416 \\
\hline & Control obese & $3.02 \pm 0.78$ & 0.010 & \\
\hline & Mangosteen obese & $2.97 \pm 0.85$ & 0.005 & 0.934 \\
\hline & Curcumin obese & $2.67 \pm 1.31$ & 0.165 & 0.628 \\
\hline & Synergistic obese & $2.78 \pm 0.67$ & 0.003 & 0.620 \\
\hline \multirow[t]{8}{*}{ HDL-C /LDL-C } & Control lean & $4.61 \pm 1.21$ & & \\
\hline & Mangosteen lean & $2.54 \pm 1.02$ & & 0.016 \\
\hline & Curcumin lean & $1.95 \pm 0.40$ & & 0.009 \\
\hline & Synergistic lean & $5.35 \pm 1.43$ & & 0.465 \\
\hline & Control obese & $1.15 \pm 0.73$ & 0.009 & \\
\hline & Mangosteen obese & $0.57 \pm 0.07$ & 0.009 & 0.346 \\
\hline & Curcumin obese & $1.21 \pm 0.79$ & 0.117 & 0.917 \\
\hline & Synergistic obese & $0.95 \pm 0.51$ & 0.009 & 1.000 \\
\hline \multirow[t]{8}{*}{ GLU (mg/dl) } & Control lean & $232.55 \pm 74.67$ & & \\
\hline & Mangosteen lean & $154.43 \pm 34.27$ & & 0.076 \\
\hline & Curcumin lean & $121.12 \pm 8.90$ & & 0.009 \\
\hline & Synergistic lean & $162.08 \pm 39.89$ & & 0.117 \\
\hline & Control obese & $206.94 \pm 100.22$ & 0.347 & \\
\hline & Mangosteen obese & $169.67 \pm 36.21$ & 0.346 & 0.917 \\
\hline & Curcumin obese & $174.43 \pm 56.07$ & 0.076 & 0.754 \\
\hline & Synergistic obese & $155.44 \pm 74.38$ & 0.917 & 0.754 \\
\hline
\end{tabular}

a Significant difference between normal weight and obese rats in each group (Control, Mangosteen, 
Curcumin, and Synergistic)

b Significant difference between each group and respective normal weight or obese control groups

Table 5

Pearson's correlation coefficients between measured variables.

\begin{tabular}{|c|c|c|c|}
\hline Parameters & $\mathrm{R}$ (Correlation Coefficient) & $\mathrm{p}$-value & Correlation \\
\hline BMI with $\mathrm{CHOL}$ & $0.601 * \star$ & 0.000 & $P^{a}$ \\
\hline BMI with HDL-C & $-0.381^{*}$ & 0.015 & $\mathrm{~N}^{\mathrm{b}}$ \\
\hline BMI with GSH & $-0.298^{*}$ & 0.011 & $N^{b}$ \\
\hline BMI with $\mathrm{CHOL} / \mathrm{HDL}-\mathrm{C}$ & $0.645^{\star \star}$ & 0.000 & $\mathrm{P}^{\mathrm{a}}$ \\
\hline CHOL with TRIG & $0.507 \star \star$ & 0.001 & $\mathrm{P}^{\mathrm{a}}$ \\
\hline TRIG with $\mathrm{CHOL} / \mathrm{HDL}-\mathrm{C}$ & $0.384^{\star}$ & 0.014 & $\mathrm{P}^{\mathrm{a}}$ \\
\hline LDL-C with BMI & 0.634 ** & 0.000 & $\mathrm{P}^{\mathrm{a}}$ \\
\hline LDL-C with GSH & $0.343^{*}$ & 0.030 & $\mathrm{~N}^{\mathrm{a}}$ \\
\hline LDL-C with $\mathrm{CHOL}$ & $0.955^{\star \star}$ & 0.000 & $\mathrm{P}^{\mathrm{a}}$ \\
\hline LDL-C with TRIG & $0.451^{\star \star}$ & 0.003 & $\mathrm{P}^{\mathrm{a}}$ \\
\hline LDL-C with HDL-C / LDL-C & $-0.927 * \star$ & 0.000 & $\mathrm{~N}^{\mathrm{b}}$ \\
\hline \multicolumn{4}{|l|}{${ }^{* *} p<0.01$} \\
\hline \multicolumn{4}{|l|}{${ }^{*} p<0.05$} \\
\hline \multicolumn{4}{|l|}{ a Positive correlation } \\
\hline${ }^{b}$ Negative correlation & & & \\
\hline
\end{tabular}


Table 6

ROC of all parameters for obese rats.

\begin{tabular}{|c|c|c|c|c|c|c|c|}
\hline & Synergistic obese & 1.000 & 27.970 & $\begin{array}{l}100.0 \\
\%\end{array}$ & $\begin{array}{l}100.0 \\
\%\end{array}$ & 0.009 & $\begin{array}{l}1.000- \\
1.000\end{array}$ \\
\hline \multirow[t]{4}{*}{ TRIG } & Control obese & 1.000 & 204.290 & $\begin{array}{l}100.0 \\
\%\end{array}$ & $\begin{array}{l}100.0 \\
\%\end{array}$ & 0.009 & $\begin{array}{l}1.000- \\
1.000\end{array}$ \\
\hline & $\begin{array}{l}\text { Mangosteen } \\
\text { obese }\end{array}$ & 0.960 & 211.535 & $\begin{array}{l}100.0 \\
\%\end{array}$ & $80.0 \%$ & 0.016 & $\begin{array}{l}0.843- \\
1.077\end{array}$ \\
\hline & Curcumin obese & 0.600 & 226.895 & $60.0 \%$ & $\begin{array}{l}100.0 \\
\%\end{array}$ & 0.602 & $\begin{array}{l}0.171- \\
1.029\end{array}$ \\
\hline & Synergistic obese & 0.800 & 242.755 & $80.0 \%$ & $\begin{array}{l}100.0 \\
\%\end{array}$ & 0.117 & $\begin{array}{l}0.449- \\
1.151\end{array}$ \\
\hline \multirow[t]{4}{*}{$\mathrm{CHOL} / \mathrm{HDL}-\mathrm{C}$} & Control obese & 1.000 & 1.950 & $\begin{array}{l}100.0 \\
\%\end{array}$ & $\begin{array}{l}100.0 \\
\%\end{array}$ & 0.009 & $\begin{array}{l}1.000- \\
1.000\end{array}$ \\
\hline & $\begin{array}{l}\text { Mangosteen } \\
\text { obese }\end{array}$ & 1.000 & 1.800 & $\begin{array}{l}100.0 \\
\%\end{array}$ & $\begin{array}{l}100.0 \\
\%\end{array}$ & 0.009 & $\begin{array}{l}1.000- \\
1.000\end{array}$ \\
\hline & Curcumin obese & 0.800 & 1.970 & $80.0 \%$ & $80.0 \%$ & 0.117 & $\begin{array}{l}0.494- \\
1.106\end{array}$ \\
\hline & Synergistic obese & 1.000 & 1.790 & $\begin{array}{l}100.0 \\
\%\end{array}$ & $\begin{array}{l}100.0 \\
\%\end{array}$ & 0.009 & $\begin{array}{l}1.000- \\
1.000\end{array}$ \\
\hline \multirow[t]{4}{*}{$\begin{array}{l}\mathrm{HDL}-\mathrm{C} / \mathrm{LDL}- \\
\mathrm{C}\end{array}$} & Control obese & 1.000 & 2.470 & $\begin{array}{l}100.0 \\
\%\end{array}$ & $\begin{array}{l}100.0 \\
\%\end{array}$ & 0.009 & $\begin{array}{l}1.000- \\
1.000\end{array}$ \\
\hline & $\begin{array}{l}\text { Mangosteen } \\
\text { obese }\end{array}$ & 1.000 & 1.065 & $\begin{array}{l}100.0 \\
\%\end{array}$ & $\begin{array}{l}100.0 \\
\%\end{array}$ & 0.009 & $\begin{array}{l}1.000- \\
1.000\end{array}$ \\
\hline & Curcumin obese & 0.800 & 1.305 & $80.0 \%$ & $\begin{array}{l}100.0 \\
\%\end{array}$ & 0.117 & $\begin{array}{l}0.449- \\
1.151\end{array}$ \\
\hline & Synergistic obese & 1.000 & 2.605 & $\begin{array}{l}100.0 \\
\%\end{array}$ & $\begin{array}{l}100.0 \\
\%\end{array}$ & 0.009 & $\begin{array}{l}1.000- \\
1.000\end{array}$ \\
\hline \multirow[t]{4}{*}{ GLU } & Control obese & 0.680 & 152.088 & $60.0 \%$ & $\begin{array}{l}100.0 \\
\%\end{array}$ & 0.347 & $\begin{array}{l}0.301- \\
1.059\end{array}$ \\
\hline & $\begin{array}{l}\text { Mangosteen } \\
\text { obese }\end{array}$ & 0.680 & 145.168 & $80.0 \%$ & $60.0 \%$ & 0.347 & $\begin{array}{l}0.328- \\
1.032\end{array}$ \\
\hline & Curcumin obese & 0.840 & 141.216 & $60.0 \%$ & $\begin{array}{l}100.0 \\
\%\end{array}$ & 0.076 & $\begin{array}{l}0.580- \\
1.100\end{array}$ \\
\hline & Synergistic obese & 0.520 & 139.069 & $80.0 \%$ & $40.0 \%$ & 0.917 & $\begin{array}{l}0.135- \\
0.905\end{array}$ \\
\hline
\end{tabular}


Table 7

Multiple regression analysis using GSH levels as the dependent variable.

\begin{tabular}{|c|c|c|c|c|c|c|}
\hline \multirow[t]{2}{*}{ Predictor Variable } & \multirow[t]{2}{*}{ Coefficient } & \multirow[t]{2}{*}{ SE } & \multirow[t]{2}{*}{$p$-value } & \multirow[t]{2}{*}{ Adjusted R2 } & \multicolumn{2}{|l|}{$95 \% \mathrm{Cl}$} \\
\hline & & & & & Lower & Upper \\
\hline LDL-C & 1.196 & 0.285 & 0.000 & 0.298 & 0.618 & 1.773 \\
\hline LDL-C & 2.355 & 0.391 & 0.983 & 0.482 & 1.563 & 3.146 \\
\hline HDL-C / LDL-C & 25.584 & 6.716 & 0.000 & & 11.976 & 39.191 \\
\hline LDL-C & 2.231 & 0.350 & 0.000 & 0.589 & 1.521 & 2.941 \\
\hline HDL-C /LDL-C & 26.558 & 5.990 & 0.000 & & 14.409 & 38.707 \\
\hline MDA & -1.509 & 0.463 & 0.002 & & -2.448 & -0.570 \\
\hline
\end{tabular}

\section{Discussion}

A diet high in fat leads to obesity in both humans and animals [22,23]. In both rats and mice, a positive relationship has been demonstrated between the levels of fat in the diet and body weight or fat gain; also, rats that consumed diets containing high quantities of fat gained weight faster than those on diets containing minimal fat [24-28].

The recorded increase of BMI in obese rats is in agreement with previous studies [29], in which they reported that BMI in obese rats is usually higher than $0.75 \mathrm{gm} / \mathrm{cm}^{2}$. The final weight and weight gain in obese rats were also coupled with high BMI, in agreement with the findings of Picklo et al [30], who showed the obesogenic effect of a saturated lipid diet in animal models. Unexpectedly, while both mangosteen pericarp extract and curcumin were effective in lowering the BMI of obese animals, both also induced weight gain in lean animals. This is similar to the findings of Husen et al [31], who reported an increase in body weight following mangosteen extract and curcumin after the decrease in body weight induced by STZ treatment.

The elevated levels of ROS after high caloric intake or inflammation can later result in increase of BMI or incidence of obesity. Adipose tissue can undergo pathological alterations induced by inflammation or oxidative stress, which in turn enhances the secretion of adipokines and affects the peripheral tissues that produce ROS, further promoting oxidative stress and the inflammatory response [32] (see also Cristancho \& Lazar 2011). The concentration of the serum MDA can be used as an indicator of oxidative stress. MDA is one of the final products of the peroxidation of polyunsaturated fatty acids (PUFA). The concentration of MDA can be used as an indicator of cell or tissue damage due to the increase in lipid peroxidation. The unexpected non-significant increase of MDA and decrease in GSH levels, GST activity, and vitamin $\mathrm{C}$ levels in HFD-induced obese rats might be attributed to the addition of coconut oil to the high saturated fat diet given in our paradigm as an inducer of obesity. Multiple studies demonstrate the anti-oxidative effects of virgin coconut oil, supporting this hypothesis $[33,34]$. Table 2 also shows the 
independent and synergistic effects of mangosteen pericarp extract and curcumin on oxidative stressrelated variables. While mangosteen pericarp extract did not demonstrate anti-oxidative effects in normal weight rats, it did induce a significant increase in GSH levels in obese rats. GST functions as antioxidant, and enzymes can catalyze the detoxification of xenobiotics via conjugation with GSH.

The diet-induced obesity animal model is one of the most common and reliable models used in obesity studies due to its similarity in modeling the most common route of obesity in humans, as well as related metabolic effects. As shown previously, this HFD models obesity via increased food intake, body weight gain, body fat accumulation, BMI increase, defects in antioxidant levels, and disruption in the lipid profile $[22,28]$.

While LDL-C is responsible for the delivery of cholesterol to peripheral tissues, HDL-C mediates the inverse process of cholesterol transport from peripheral tissues [35]. The non-significant decrease of HDL-C in obese rats reported in the present study may be related to the anti-oxidative effect of coconut oil, a component of the HFD used here [33,34].

In general, ingestion of coconut oil can increase HDL-C [36]. It has been proposed that lauric acid, the main constituent of coconut oil, is the cornerstone of this pathway. Lauric acid accounts for $50 \%$ of the content of coconut oil. Although lauric acid is considered a medium-chain fatty acid (MCFA), 70\% of lauric acid is transported as a long-chain fatty acid (LCFA), while the other $30 \%$ remains as a MCFA. Thus, there are two ways of transporting lauric acid in the body. When lauric acid reaches the liver, it serves as a substrate in the production of apoA1 and apoB, further contributing to the formation of both HDL-C and LDL-C [36].

Studies show that in contrast, to LDL-C, HDL-C may play an anti-atherogenic and anti-thrombotic role by protecting LDL-C particles against lipid peroxidation and reducing the deleterious effects of oxidized LDL$C$ [37]. Based on this report, the non-significant decrease of HDL-C in obese rats may be related to the presence of coconut oil in the HFD [38].

Moreover, our data is consistent with those of Wihastuti et al [39], who showed that $400 \mathrm{mg} / \mathrm{kg}$ body weight mangosteen pericarp extract affected LDL-C but no other lipid marker, and significantly reduced $\mathrm{H}_{2} \mathrm{O}_{2}$ levels and NF-KB expression. At concentrations of $800 \mathrm{mg} / \mathrm{kg}$ body weight, this extract was most effective in improving the lipid profile; this suggests that although in the present study mangosteen pericarp extract did induce a hypo-lipidemic effect in obese rats, the anti-atherogenic effects likely occurred via its anti-oxidative and anti-inflammatory effects [39].

This contradiction may also be attributed to the fact the amount of total xanthone in the mangosteen pericarp extract is strongly affected by the extraction capacity of the solvent to recover different phenolic constituents from various fruit origins, as well as the methods of transportation and storage [40-42]. Aisha, Abu-Salah \& Ismail [43] have reported that toluene is the most efficient extraction solvent for mangosteen pericarp extract compared to $75 \%$ ethanol and methanol [44]. 
Obesity is a risk factor for the development of cardiovascular disease, diabetes mellitus, hyperlipidemia, and arteriosclerosis (Cannon, 2007). To treat and prevent obesity and obesity-related complications, an increasing number of people use hypoglycemia-inducing or weight-loss drugs. However, the long-term use of these drugs can damage the liver and kidney. Unsurprisingly, finding safe and effective weight-lossand hypoglycemia-inducing agents is becoming increasingly urgent.

Table 4 reveals the level of serum glucose in the studied rats. While obesity itself did not induce elevation of blood glucose, mangosteen pericarp extract was effective in reducing glucose levels in both normal weight and obese animals. This is consistent with the previous work of Taher et al [45], which demonstrated that orally administered mangosteen pericarp extract at various doses demonstrated a hypoglycemic effect in streptozotocin (STZ)-induced diabetic rats and normoglycemic rats. Moreover, curcumin demonstrates hypoglycemic effects in both obese and normal weight rats; in obese rats, the synergistic effects of both of these extracts was much higher than each extract independently. This is also supported by Rivera-Mancía et al [46] and Sohaei et al [47], who showed the hypoglycemic effects of curcumin. As previously described, the use of curcumin in vitro and in animal models of diabetes revealed a variety of potential mechanisms of action to treat diabetes mellitus; however, clinical trials in humans have thus far been inconsistent with these findings.

Pearson's correlation coefficient (PCC) is a statistical metric that measures the strength and direction of a linear relationship between two or more random variables [48]. Table 5 indicates the correlations between all variables measured in this study. It can be seen that obesity (BMI), antioxidant status (GSH levels), and dyslipidemia-related markers (CHOL, TAG, CHOL/HDL-C) were negatively or positively correlated in a manner that demonstrates the negative impact of oxidative stress and dyslipidemia on obesity.

Abruzzo et al [49] highlighted the advantage of using ROC curves as an outstanding statistical tool for the identification of biomarkers that are sufficiently sensitive and specific for the early diagnosis of obesity. Although its utility in prediction, risk valuation, and assessment of therapeutic interventions still requires further validation, ROC curves emphasize the most significant statistical differences between patients and controls and even animal models of diseases [50]. The AUC provides a useful measure to evaluate the predictive value of biomarkers. While an AUC value near 1 designates an excellent predictive marker, a curve that lies adjacent to the diagonal (AUC $=0.5$ ) indicates no diagnostic usefulness. AUC values close to 1.00 are always accompanied by satisfactory values of specificity and sensitivity [51].

Table 6 demonstrates the ROC curves with AUC, specificity, and sensitivity in obese rats. Among the measured variables, BMI showed excellent predictive value as a marker of obesity, with AUC between 0.8 and 1 with satisfactory specificity and sensitivity. The other measured variables demonstrate relatively less predictive ability with any specificity and sensitivity.

\section{Conclusion}

The present study ascertained the efficiency of a HFD in inducing obesity in rats, and the effectiveness of mangosteen pericarp extract and curcumin independently or synergistically in treating obesity-induced 
atherogenesis.

\section{Declarations \\ Conflict of interest}

The authors declare no potential conflicts of interest with respect to the authorship, and/or publication of this article.

\section{Acknowledgments}

The authors thank the Deanship of Scientific Research for funding and supporting this research through the DSR Graduate Students Research Support (GSR) initiative. The authors also extend their gratitude to the RSSU at King Saud University for their technical support.

\section{Funding}

This work was supported by the Deanship of Scientific Research through the DSR Graduate Students Research Support (GSR) initiative.

\section{Author's Contribution}

RL: Aquistion of the data

HA: Supervise the practical work

AA: Performing the experimental work

MA: Co-drafted the manuscript

RB: Aquistion of the data

AE: Supervise the experimental work and drafted the manuscript

All authors reviewed the manuscript.

\section{References}

1. Manna, P. \& Jain, S. K. Obesity, oxidative stress, adipose tissue dysfunction, and the associated health risks: causes and therapeutic strategies. Metab Syndr Relat Disord. 13 (10), 423-444 https://doi.org/10.1089/met.2015.0095 (2015). 
2. Scherer, P. E. \& Hill, J. A. Obesity, diabetes, and cardiovascular diseases. Circ Res. 118 (11), 17031705 https://doi.org/10.1161/CIRCRESAHA.116.308999 (2016).

3. Boccellino, M. et al. AT1-receptor blockade: protective effects of irbesartan in cardiomyocytes under hypoxic stress. PLOS ONE. 13 (10), e0202297 https://doi.org/10.1371/journal.pone.0202297 (2018).

4. Vincent, H. K. \& Taylor, A. G. Biomarkers and potential mechanisms of obesity-induced oxidant stress in humans. Int J Obes. 30 (3), 400-418 https://doi.org/10.1038/sj.ijo.0803177 (2006).

5. Vanacore, D. et al. Effect of restriction vegan diet's on muscle mass, oxidative status, and myocytes differentiation: A pilot study. J Cell Physiol. 233 (12), 9345-9353 https://doi.org/10.1002/jcp.26427 (2018).

6. Akbari, F. \& Azadbakht, L. A systematic review on diet quality among Iranian Youth: focusing on reports from Tehran and Isfahan. Arch Iran Med. 17(8):574-584(2014) doi: 014178/AIM.0010. .

7. Azadbakht, L., Akbari, F. \& Esmaillzadeh, A. Diet quality among Iranian adolescents needs improvement. Public Health Nutr. 18 (4), 615-621 https://doi.org/10.1017/S1368980014000767 (2015).

8. Rothwell, N. J. \& Stock, M. J. The development of obesity in animals: the role of dietary factors. Clin Endocrinol Metab. 13 (3), 437-449 https://doi.org/10.1016/s0300-595x(84)80032-8 (1984).

9. Pandita, A. et al. Childhood obesity: prevention is better than cure. Diabetes Metab Syndr Obes Targets Ther. 9, 83-89 https://doi.org/10.2147/DMSO.S90783 (2016).

10. Aizat, W. M. et al. Recent updates on metabolite composition and medicinal benefits of mangosteen plant. PeerJ. 7, e6324 https://doi.org/10.7717/peerj.6324 (2019).

11. Lin, C. Y. et al. Anticoagulatory, antiinflammatory, and antioxidative effects of protocatechuic acid in diabetic mice. J Agric Food Chem. 57 (15), 6661-6667 https://doi.org/10.1021/jf9015202 (2009).

12. Chae, H. S. et al. Mangosteen extract prevents dextran sulfate sodium-induced colitis in mice by suppressing NF-KB activation and inflammation. J Med Food. 20 (8), 727-733 https://doi.org/10.1089/jmf.2017.3944 (2017).

13. Mamat, S. F. et al. Metabolomics analysis of mangosteen (Garcinia mangostana Linn.) fruit pericarp using different extraction methods and GC-MS. Plant OMICS. 11 (2), 89-97 https://doi.org/10.21475/poj.11.02.18.pne1191 (2018).

14. Chainani-Wu, N. Safety and anti-inflammatory activity of curcumin: A component of tumeric (Curcuma longa). J Altern Complement Med. 9 (1), 161-168 https://doi.org/10.1089/107555303321223035 (2003).

15. Pierro, F. et al. Potential role of bioavailable curcumin in weight loss and omental adipose tissue decrease: preliminary data of a randomized, controlled trial in overweight people with metabolic syndrome. Preliminary study. Eur Rev Med, (19). Eur Rev Med Pharmacol Sci. 19 (21), 4195-4202 (2015).

16. Ghazimoradi, M. et al. The effects of curcumin and curcumin-phospholipid complex on the serum pro-oxidant-antioxidant balance in subjects with metabolic syndrome. Phytother Res. 31 (11), 1715-1721 https://doi.org/10.1002/ptr.5899 (2017). 
17. Sasidharan, S. R. et al. An experimental approach for selecting appropriate rodent diets for research studies on metabolic disorders. BioMed Res Int. 2013, 1-9 https://doi.org/10.1155/2013/752870 (2013).

18. Begonaruizlarrea, M. et al. Antioxidant effects of estradiol and 2-hydroxyestradiol on iron-induced lipid peroxidation of rat liver microsomes. Steroids. 59 (6), 383-388 https://doi.org/10.1016/0039128X(94)90006-X (1994).

19. Jagota, S. K. \& Dani, H. M. A new colorimetric technique for the estimation of vitamin $C$ using Folin phenol reagent. Anal Biochem. 127 (1), 178-182 https://doi.org/10.1016/0003-2697(82)90162-2 (1982).

20. Beutler, E., Duron, O. \& Kelly, B. M. Improved methods for determination of blood Gluthatione. J Lab Clin Med. 61, 882-888 (1963).

21. Habig, W. H. \& Pabst, M. J. JW. Glutathione S-transferases. The first enzymatic step in mercapturic acid formation. J Biol Chem. 25 (22), 249 (1974).

22. Buettner, R., Schölmerich, J. \& Bollheimer, L. C. High-fat diets: modeling the metabolic disorders of human obesity in rodents. Obesity. 15 (4), 798-808 https://doi.org/10.1038/oby.2007.608 (2007).

23. Warwick, Z. S. \& Schiffman, S. S. Role of dietary fat in calorie intake and weight gain. Neurosci Biobehav Rev. 16 (4), 585-596 https://doi.org/10.1016/s0149-7634(05)80198-8 (1992).

24. Bourgeois, F., Alexiu, A. \& Lemonnter, D. Dietary-induced obesity: effect of dietary fats on adipose tissue cellularity in mice. Br J Nutr. 49 (1), 17-26 https://doi.org/10.1079/BJN19830006 (1983).

25. Boozer, C. N. et al. Dietary fat and adiposity: a dose-response relationship in adult male rats fed isocalorically. Am J Physiol Endocrinol Metab. 268 (4), E546-E550 https://doi.org/10.1152/ajpendo.1995.268.4.E546 (1995).

26. Takahashi, M., Ikemoto, S. \& Ezaki, O. Effect of the fat/carbohydrate ratio in the diet on obesity and oral glucose tolerance in C57BL/6J mice. J Nutr Sci Vitaminol Tokyo. October. 45 (5), 583-593 https://doi.org/10.3177/jnsv.45.583 (1999).

27. Ghibaudi, L. et al. Fat intake affects adiposity, comorbidity factors, and energy metabolism of Sprague-Dawley rats. Obes Res. 10 (9), 956-963 https://doi.org/10.1038/oby.2002.130 (2002).

28. Hariri, N. \& Thibault, L. High-fat diet-induced obesity in animal models. Nutr Res Rev. 23 (2), 270-299 https://doi.org/10.1017/S0954422410000168 (2010).

29. Novelli, E. L. B. et al. Anthropometrical parameters and markers of obesity in rats. Lab Anim. 41 (1), 111-119 https://doi.org/10.1258/002367707779399518 (2007).

30. Picklo, M. J. et al. Comparative effects of high oleic acid vs high mixed saturated fatty acid obesogenic diets upon PUFA metabolism in mice. Prostaglandins Leukot Essent Fatty Acids. 119(November(2016):25-37 (2017). doi: 10.1016/j.plefa.2017.03.001. .

31. Husen, S. A. et al. Activity assay of mangosteen (Garcinia mangostana L.) pericarp extract for decreasing fasting blood cholesterol level and lipid peroxidation in type-2 diabetic mice. AIP Conf Proc. 2017;1888. doi: 10.1063/1.5004303. 
32. Kenneth, $\mathrm{H}$. et al. Therapeutic applications of reactive oxygen and nitrogen species in human disease. Free Radic Biol Med. 28 (10), 1456-1462 https://doi.org/10.1016/S0891-5849(00)00254-9 (2000).

33. Durašević, S. et al. The protective role of virgin coconut oil on the alloxan-induced oxidative stress in the liver, kidneys and heart of diabetic rats. Food Funct. 10 (4), 2114-2124 https://doi.org/10.1039/C9F000107G (2019).

34. Famurewa, A. C. et al. Antioxidant, anti-inflammatory, and antiapoptotic effects of virgin coconut oil against antibiotic drug gentamicin-induced nephrotoxicity via the suppression of oxidative stress and modulation of iNOS/NF-KB/caspase-3 signaling pathway in Wistar rats. J Food Biochem. 44 (1), (August):1-10 https://doi.org/10.1111/jfbc.13100 (2020).

35. Zhang, Y. Z. et al. Overexpression of apolipoprotein A-I promotes reverse transport of cholesterol from macrophages to feces in vivo. Circulation. 108 (6), 661-663 https://doi.org/10.1161/01.CIR.0000086981.09834.E0 (2003).

36. Santos, H. O. et al. Coconut oil intake and its effects on the cardiometabolic profile-A structured literature review. Prog Cardiovasc Dis. 62 (5), 436-443 https://doi.org/10.1016/j.pcad.2019.11.001 (2019).

37. Navab, M. et al. Thematic review series: The Pathogenesis of Atherosclerosis The oxidation hypothesis of atherogenesis: the role of oxidized phospholipids and HDL. J Lipid Res. 45 (6), 9931007 https://doi.org/10.1194/jlr.R400001-JLR200 (2004).

38. Eyres, L. et al. Coconut oil consumption and cardiovascular risk factors in humans. Nutr Rev. 74 (4), 267-280 https://doi.org/10.1093/nutrit/nuw002 (2016).

39. Wihastuti, T. A. et al. Study of the inhibition effect of ethanolic extract of mangosteen pericarp on atherogenesis in hypercholesterolemic rat. Asian Pac J Trop Dis. 5 (10), 830-834 https://doi.org/10.1016/S2222-1808(15)60940-9 (2015).

40. Suttirak, W. \& Manurakchinakorn, S. In vitro antioxidant properties of mangosteen peel extract. J Food Sci Technol. 51 (12), 3546-3558 https://doi.org/10.1007/s13197-012-0887-5 (2014).

41. Kusmayadi, A. et al. The effect of solvents and extraction time on total xanthone and antioxidant yields of mangosteen peel (Garcinia mangostana L.) extract. Drug Invent Today. 10 (12), 2572-2576 (2018).

42. Sungpud, C. et al. Tuning of virgin coconut oil and propylene glycol ratios for maximizing the polyphenol recovery and in vitro bioactivities of mangosteen (Garcinia mangostana L.) pericarp. Process Biochem. 87 (July), 179-186 https://doi.org/10.1016/j.procbio.2019.08.023 (2019).

43. Aisha, A. F. A., Abu-Salah Khalid, M., Ismail, Z., And \& Majid, A. M. S. A. Med Plants Research. Determination of total xanthones in Garcinia mangostana fruit rind extracts by ultraviolet (UV) spectrophotometry;7(1):29-35. (2013) doi: 10.5897/JMPR11.1183.

44. Mohammad, N. A. et al. Optimization of the antioxidant-rich xanthone extract from mangosteen (Garcinia mangostana L.) pericarp via microwave-assisted extraction. Heliyon. 5 (10), e02571 https://doi.org/10.1016/j.heliyon.2019.e02571 (2019). 
45. Taher, M. et al. Hypoglycaemic activity of ethanolic extract of Garcinia mangostana Linn. in normoglycaemic and streptozotocin-induced diabetic rats. BMC Complement Altern Med. 16 (1), 135 https://doi.org/10.1186/s12906-016-1118-9 (2016).

46. Rivera-Mancía, S., Trujillo, J. \& Chaverri, J. P. Utility of curcumin for the treatment of diabetes mellitus: evidence from preclinical and clinical studies. J Nutr Intermed Metab. 14, 29-41 https://doi.org/10.1016/j.jnim.2018.05.001 (2018).

47. Sohaei, S. et al. The effects of curcumin supplementation on glycemic status, lipid profile and hsCRP levels in overweight/obese women with polycystic ovary syndrome: A randomized, double-blind, placebo-controlled clinical trial. Complement Ther Med. $\mathbf{4 7}$ (September), 102201 https://doi.org/10.1016/j.ctim.2019.102201 (2019).

48. Lee Rodgers, J. \& Nicewander, W. A. Thirteen ways to look at the correlation coefficient. Am Stat. 42 (1), 59-66 https://doi.org/10.1080/00031305.1988.10475524 (1988).

49. Abruzzo, P. M. et al. Perspective biological markers for autism spectrum disorders: advantages of the use of receiver operating characteristic curves in evaluating marker sensitivity and specificity. Dis Markers. 2015(Cdc):1-16 (2015). doi: 10.1155/2015/329607. .

50. El-Ansary, A., Hassan, W. M., Daghestani, M., Al-Ayadhi, L. \& Ben Bacha, A. Preliminary evaluation of a novel nine-biomarker profile for the prediction of autism spectrum disorder. PLOS ONE. 15 (1), e0227626 https://doi.org/10.1371/journal.pone.0227626 (2020).

51. Metz, C. E. Basic principles of ROC analysis. Semin Nucl Med. 8 (4), 283-298 https://doi.org/10.1016/s0001-2998(78)80014-2 (1978). 